\title{
Impurity Effect on the Two-Dimensional-Electron Fluid-Solid Transition in Zero Field
}

\author{
S. T. Chui ${ }^{1}$ and B. Tanatar ${ }^{2}$ \\ ${ }^{1}$ Bartol Research Institute, University of Delaware, Newark, Delaware 19716 \\ ${ }^{2}$ Physics Department, Bilkent University, 06433 Ankara, Turkey
}

(Received 15 June 1994)

\begin{abstract}
We investigate the effect of impurities on the electron fluid-solid transition with parameters appropriate for the system recently studied by Pudalov et al. The nature of the crystalline state at $T=0$ in the presence of impurities is studied with the relaxation technique. The solid-fluid transition is studied via perturbation calculation and Monte Carlo simulation. The transition density is found to shift from $r_{s} \approx 37$ for the pure system to $r_{s} \approx 7.5$, close to that observed experimentally. At this small value of $r_{s}$, the fluid energy is sensitive to the spin polarization but the solid is not, suggesting possible interesting magnetic behavior.
\end{abstract}

PACS numbers: 73.40.Lq, 72.20.Ht, 72.20.My, 72.70.+m

Recently there has been much interest in the low density limit of 2D electrons in GaAs heterojunctions in an external magnetic field [1] and Si-MOSFET's in high field where a freezing transition to a solid seems to occur as the density is lowered.

Monte Carlo (MC) [2] and analytic calculations [3] for the pure system at zero magnetic field suggest that the solid-fluid transition occurs near $r_{s} \approx 37$. Here $r_{s}=$ $1 / \sqrt{\pi n} a_{B}$, where $a_{B}=\hbar^{2} \epsilon / m^{*} e^{2}$ is the Bohr radius, $n$ is the density, $m^{*}(0.2 \mathrm{~m}$ for Si-MOSFET's) is the effective mass, and $\epsilon$ (7.7 for Si-MOSFET's) is the dielectric constant. Recently Pudalov et al. [4] reported observation of a fluid-solid transition in Si-MOSFET's near $r_{s}=10$. The experiential systems are not perfect. To confront experiment with theory, a quantitative calculation that includes the effect of both electron-electron interaction and external defects is essential. In this Letter we study impurity on the electron fluid and solid with parameters appropriate for the Si-MOSFET system investigated by Pudalov et al.

We study the nature of the classical crystalline state at $T=0$ in the presence of impurities by seeking the lowest energy configuration numerically with the quasiNewton method [5]. This follows our earlier study of impurity effects on the GaAs heterostructures [6], where we found the relaxation due to the impurities to be well approximated by perturbation theory, the relaxation being mainly longitudinal in nature. In the present case, the main impurities are the $\mathrm{Na}^{+}$ions. The distance between the impurities to the electrons divided by the Bohr radius are here three times smaller than in the GaAs heterostructures. We found that perturbation results are no longer quantitatively accurate, but the average relaxation is still $60 \%$ longitudinal in nature. In earlier studies of impurity pinning [7], the longitudinal mode is completely ignored. Examples of the relaxed state at different $r_{s}$ and different impurity positions are shown in Fig. 1. Close to the solid-fluid transition near $r_{s}=7.5$ the system is quite crystalline. It rapidly becomes quite amphorous at $r_{s}=20$.
The solid-fluid transition is studied via perturbation calculation and Monte Carlo simulation. With both methods, the solid-fluid transition is found to shift from $r_{s} \approx 37$ for the pure system to $r_{s} \approx 7.5$, close to the observed experimental results. The dominant driving force behind the phase transition seems to be the following: In the solid phase, it is easier to adjust to the impurities; the difference between the impurity energies in the solid and fluid phase compensated for the energy difference between the solid and the fluid phase. While the perturbation calculation is quantitatively inaccurate, it provides valuable insight; the overall magnitude of this result and its density dependence are consistent with the quantum Monte Carlo results.

While at $r_{s}=37$ the energy of fluid and solid phases of different polarizations are very close to each other, at the small value of $r_{s} \approx 7.5$ where the transition now occurs, just as in the pure case the fluid energy is sensitive to the
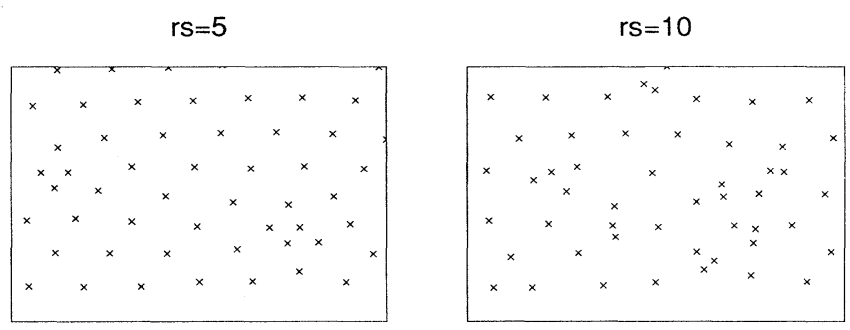

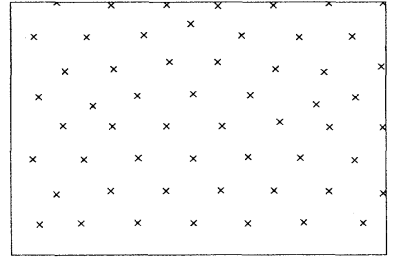

$\mathrm{rs}=7.5$

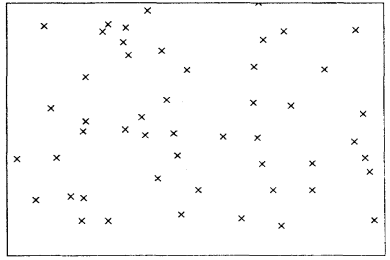

$r s=20$
FIG. 1 The relaxed lattice position at different $r_{s}$ for samples with 56 particles under periodic boundary conditions.

(C) 1995 The American Physical Society 
spin polarization but the solid is not, suggesting possible interesting magnetic behavior accompanying the solidfluid transition. We now explain our results in detail.

The Hamiltonian of our system is the sum of the kinetic energy, the interparticle Coulomb interaction, and the external impurity potential. The external potential comes from [8] surface roughness and $\mathrm{Na}^{+}$charged impurities $\left(V_{d}\right)$ of concentration [4] of approximately $10^{10} \mathrm{~cm}^{-2}$ randomly placed at positions $R_{j}$ in the $x-y$ plane at a distance $d$ of $100 \AA$ from the electrons. The Fourier transform of $V_{d}$ is given by $V_{d}(q)=\sum_{j} \exp \left(i \mathbf{q} \cdot \mathbf{R}_{j}\right) U(q)$, where $U(q)=-2 \pi e^{2} \exp (-q d) / \epsilon q$ is the Fourier transform of the Coulomb potential. We have done calculations with and without the surface roughness and found that the impurity energy changes by less than $5 \%$ at $r_{s}=7.5$. We thus focus on $V_{d}$ from now on.

We first discuss the classical crystalline state at $T=0$ in the presence of impurities. We generate samples of random positions of the impurities of a given density. For each impurity configuration we look for the local minimum for the sum of the total interelectron Coulomb potential and the impurity energy, starting with an initial configuration of a crystalline state [9]. This minimization is achieved with the standard quasi-Newton algorithm. The calculation is done under periodic boundary conditions. We have extended the Ewald sum technique to deal with the long range nature of the impurity potential [10]. Examples of the final configuration at different electron densities are shown in Fig. 1. These results suggest the following qualitative physical picture. The impurity density is fixed. At small $r_{s}$, the number of impurities per electron is small. For those electrons near an impurity, they move by a substantial amount and quickly "screen" it out. The resulting relaxation seems to be localized around the impurity. (See the graph for $r_{s}=5$.) As $r_{s}$ is increased, the number of impurities per electron is increased. The system becomes "amorphous" when an effective "percolation threshold" is reached, when the patches of local disturbance form a continuous network. While near melting near $r_{s} \approx 7.5$, the system seems quite crystalline; this crystallinity is quickly lost so that at $r_{s}=20$, the system looks quite random.

In the related problem of flux lattice melting [11], dislocations are found to be induced by impurities in 2D [12]. For our choice of parameters and sample size, we do not observe impurity induced dislocations at $r_{s}=7.5$. We think the difference lies in the softer interparticle potential (which is logarithmic in character) and a bigger and more rapidly varying impurity force for the flux line lattices. On the other hand, dislocation may be present near $r_{s}=20$, suggesting a threshold for its generation. Dislocation pairs are also observed even in the pure system as a result of quantum fluctuations [13].

We have recently studied [6] impurity effects in the GaAs system where the dopants are farther away. We found that Fourier transform of the relaxation is mainly longitudinal in nature and well approximated by perturbation theory. In the present case, the impurities are closer; perturbation results are no longer quantitatively accurate. For each sample we compute the deviation of the lattice position and its longitudinal $(l)$ and transverse $(t)$ Fourier transforms $\delta \vec{r}_{q j}=\sum_{i}\left(\mathbf{r}_{i}-\mathbf{r}_{i 0}\right) \mathbf{e}_{q j} e^{i \mathbf{q} \cdot \mathbf{r}_{i 0}} / \sqrt{N}$. Here $\mathbf{e}_{q j}$ is the polarization vector for mode $j=l, t$. We found that the relaxation is still $60 \%$ longitudinal in nature. Earlier studies of pinning [7] have ignored the longitudinal mode.

We next turn to a simple perturbation calculation for the fluid and solid energies in the presence of impurities. This calculation provides for physical insight into how impurities can lower the energy of the solid phase. The energy change $E_{i}$ of the electron system due to an external potential $V_{i}$ in linear response is given by $-0.5 \sum_{q} \chi(q) V_{i}(q)^{2} / n$, where $\chi$ is the response function. In the solid phase, in the harmonic approximation, the response function is given by $\chi_{\text {solid }}(q+G)=\sum_{i} \mid(q+$ $G)\left.e_{q i}\right|^{2} / m \omega_{q i}^{2}$ [14]. In the fluid phase, we approximate the response function with the random phase approximation with the Hubbard correction. We show in Fig. 2(a) the difference in this impurity energy between the solid and the fluid phase, $\Delta E_{i}$, together with the pure solid and fluid energy differences at different densities. As we see from the solid line representing the total energy difference, the solid phase is favored for $r_{s}>8$. Because of the localized

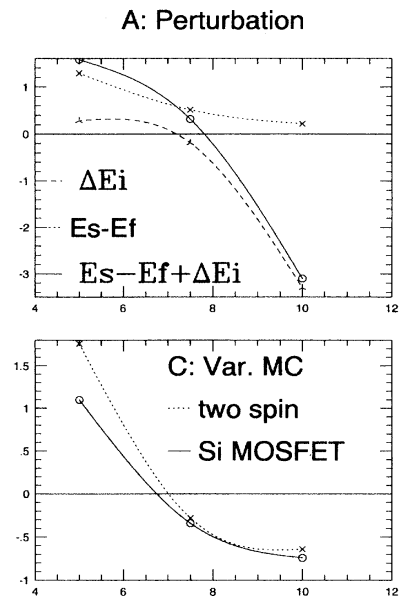

rs
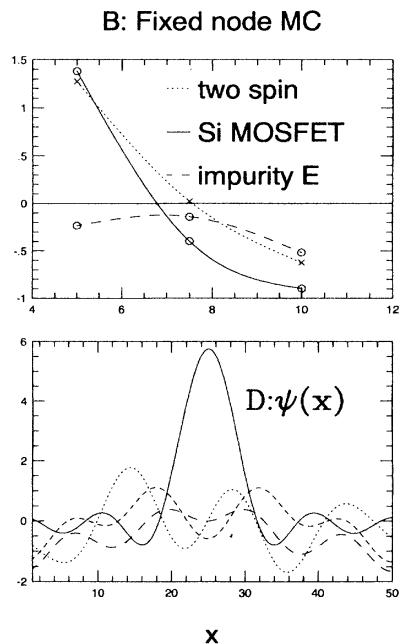

FIG. 2. (a) The contributions to the difference in the energy per particle between the solid and the fluid in units of $10^{-2} \mathrm{Ry}$ as a function of density parameter $r_{s}$. The lines are a spline fit to guide the eye. (b) The difference between solid and fluid fixed-node $\mathrm{MC}$ energies per particle, $E_{s}-E_{f}$, in units of $10^{-2} \mathrm{Ry}$, as a function of the density parameter $r_{s}$ for fluids with different degrees of polarization. The lines are spline fits through the points to guide the eye. (c) Same as (a), except that the results are for variational calculations. (d) Single-particle impurity wave functions at energies from the Fermi level to the bottom of the band at $y=0$ with $x$ from one to the other side of the box. The unit of length is such that the box length in the $x$ direction is 50 . 
nature of the solid, $\chi_{\text {solid }}>\chi_{\text {liquid }}$ at large momentum transfers; it is easier to take advantage of the impurities at low densities. $\Delta E_{i}$ becomes larger in magnitude than the energy difference between the solid and the fluid phase and thus stabilizes the solid at the transition point. While we think this calculation captures the essential physics, it may not be quantitatively very accurate. We thus turn to quantum Monte Carlo calculations.

In the variational calculation, one starts with a trial wave function $\Psi$ and calculates the expectation value of the Hamiltonian $\langle\Psi|H| \Psi\rangle$ with a Monte Carlo method. In the fixed-node calculation, one starts with the trial wave function as an initial state, then solves the time dependent Schrodinger equation assuming that the position of the node of the wave function remains unchanged. The trial wave function $\Psi_{a}(a=f$ or $s$ for fluids or solids) for the pure system is a product of a Slater determinant $D_{a}(r)$ and a Jastrow factor, $\Psi_{a}=$ $D_{a}(r) \exp \left[-\sum_{i<j} u_{a}\left(r_{i j}\right)\right]$. For the fluid, $D_{f}$ is a Slater determinant of plane waves. For the solid $D_{s}(r)$ is a determinant of Gaussian orbitals $\exp \left[-C(r-R)^{2}\right]$ localized at regular lattice sites. The Fourier transform of the solid phase pseudopotential is $2 \hat{u}_{s}(k)=-1-4 C^{\prime} / k^{2}+(1+$ $\left.8 C^{\prime} / k^{2}+4 m v(k) / \hbar^{2} k^{2}\right)^{0.5} . \quad C$ is set equal to $C^{\prime}$ in the pure system. A table of $C$ as a function of $r_{s}$ is given in Ref. [14].

For the fluid trial wave functions in the presence of the external impurities, we have solved for the single-particle wave function in the presence of the screened impurity potential $U^{\prime}(q)=-2 \pi e^{2} \exp (-q d) /\left(q \epsilon_{H}\right)$, where $e_{H}$ is the Hubbard approximation to the screening function. The product of a Slater determinant of these impurity single-particle wave functions and the Jastrow factor of the pure system is our starting point for the fixednode MC calculation. This choice of the trial function represents a good approximation for the position of the nodes. We have also performed fixed-node MC calculations starting with the trial wave function for the pure system and found the energy obtained to be indeed higher.

The solid trail wave function in the presence of impurities is formed from a Slater determinant of Gaussian orbitals located at the equilibrium sites described earlier combined with the Jastrow factor for the pure solid. It is believed $[6,15]$ that an average gap develops for the phonon modes in the presence of impurities. Thus the constant $C$ in the Gaussians need not be equal to the $C^{\prime}$ in the Jastrow factor. We have experimented with making them different and found that the difference is beyond the accuracy of our calculation. We found, however, that a $C$ that is higher than that of the pure system by $10 \%$ generally provided for a slightly lower energy.

In our simulation, the impurity potential is tabulated over a $200 \times 200$ mesh inside the box and interpolated in between. We averaged over 10 impurity samples for the different phases at different densities.
In Figs. 2(b) and 2(c), we show the average difference between the solid and the fluid energies per particle $\left(E_{s}-\right.$ $E_{f}$ ) for fluids with two spin components for fixed-node [Fig. 2(b)] and variational [Fig. 2(c)] MC calculations. The solid-fluid transition now occurs near $r_{s}=7.5$. This is consistent with recent experimental results of Pudalov et al. [4], who observed transport anomalies suggestive of a freezing transition in (100) Si-MOSFET's. The fluctuation in the difference in energy between the solid and the fluid is much smaller than the fluctuation of the total energy. For $r_{s}=7.5$, the root mean squared fluctuation of the energy difference is $2 \times 10^{-4} \mathrm{Ry}$. The errors at other densities are comparable. In contrast to the pure system, the total energy difference changes quite rapidly near the transition region, thus the demand on the accuracy of the energy is much less than that for the pure system. The impurity concentration is big enough that the transition point is shifted from $r_{s}=37$ to $r_{s}=7.5$, an experimentally accessible region.

At these higher densities the fluid energy depends on the polarization even though the solid energy is still very insensitive to the polarization. In (100) Si-MOSFET's, the electrons occupy in momentum space two valleys that are split by $2.4 \mathrm{~K}$ [16]. In addition, extrapolations of results at finite field to zero field suggest that even in zero external magnetic field the spin-up and spindown bands are split by $\approx 4 \mathrm{~K}$ in Si-MOSFET's [16] and $0.09-0.36 \mathrm{~K}$ in GaAs heterojunctions [17]. In the fluid at $r_{s}=7.5$, this splitting is comparable to the Fermi energy of $6-7.3 \mathrm{~K}$. To illustrate possible effects on the polarization we have performed calculations for a partially polarized fluid $(29,21,9$, and 0 particles for the 4 components) so that within the constraint of the finite size system, the Fermi energies of the different spinvalley manifold are as close to each other as possible after the energy splitting has been incorporated. The result for this case is also shown in Figs. 2(b) and 2(c). The transition density is shifted slightly in this case. Because of the splittings of the spin-valley manifolds we expect the solid energy to be lowest when the lowest energy spin-valley manifold is occupied, i.e., the solid is fully polarized. On the other hand, for the fluid state, the kinetic energy favors occupancy of the four spin-valley manifolds.

We next turn to the physics of the transition. According to Wigner [18], the potential energy gained due to the formation of a solid, of the order of $1 / r_{s}$, outweighs the kinetic energy lost, of the order of $1 / r_{s}^{2}$ for a low density. At low densities the energy difference between the pure solid and fluid is quite small and decreasing over a wide range of densities. The contributions to the difference in energy from the impurity potential is also shown in Figs. 2(a)-2(c). For a constant density of external defects, the energy gained from the external potential does not decrease as the electron density is decreased and eventually dominates as the density approaches zero. The 
significant amount of impurity energy suggests that the driving force for the formation of a solid is not entirely due to the Wigner mechanism.

There has been some question of the importance of localization. The $y=0$ section of five representative single-particle wave functions $\psi(x)$ in the presence of external impurities at energies from the Fermi level to the bottom of the band at $r_{s}=7.5$ is shown in Fig. 2(d). The localization length [19], if any, is much bigger than the box size of our simulation. As $r_{s}$ is increased, the impurity effect does get bigger. Localization does not seem to be a big driving force for the transition at $r_{s}=$ 7.5 , however.

While the solid phase does not have long range order in the presence of impurities [20], there can still be a difference between the solid and the fluid. For example, as we learn from past studies of finite temperature melting [21], the solid-fluid transition is connected with the absence of a shear modulus and is not directly related to the presence of long range order.

The possibility of an electron glass [19] and a Mott transition [22] has been discussed in the literature. These studies are connected with the possibility of the impurity bound states forming a band. While the physics of the solid formation is obviously related, there is one very important difference. At $r_{s}=7.5$, the impurity density is $1 / 14$ that of the electron density so that it is not possible for each electron to reside on an impurity bound state. At lower densities, these scenarios become important. The variational wave function discussed here may provide a different perspective to address the physics.

In conclusion, we have studied a model that reflects the impurity effects in (100) Si-MOSFET's and found that the solid-fluid transition can be shifted to experimentally accessible regions. The solid may be fully polarized, which could be tested experimentally. The transition $r_{s}$ obtained here is slightly lower than the experimental results. The experimental impurity concentration is deduced indirectly from transport measurements and thus approximate. For this reason we consider the agreement between theory and experiment reasonable.

We thank V. Pudalov for helpful information and for providing very useful references and D.C. Tsui for emphasizing to us the importance of external defects. This work is supported in part by NATO Grant No. CRG920487.
[1] For a recent review, see, Physics of the 2D Quantum Electron Solid, edited by S.-T. Chui (International Press, Cambridge, MA, 1994).

[2] B. Tanatar and D. Ceperley, Phys. Rev. B 39, 5005 (1989); private communication.

[3] S. T. Chui and K. Esfarjani, Europhys. Lett. 14, 361 (1991).

[4] V.M. Pudalov, M. D'Iorio, S. V. Kravchenko, and J. W. Campbell, Phys. Rev. Lett. 70, 1866 (1993).

[5] We use the quasi-Newton algorithm discussed, for example, by D. Kahaner et al., in Numerical methods and software (Prentice Hall, Englewood Cliffs, NJ, 1989), Chap. 9, subroutine UNCMND.

[6] S. T. Chui, J. Phys. Condens. Matter 5, L405 (1993).

[7] H. Fukuyama and P. A. Lee, Phys. Rev. B 18, 6245 (1978).

[8] T. Ando, A. B. Fowler, and F. Stern, Rev. Mod. Phys. 54, 449 (1982).

[9] The quantities of interest are $\sum_{q} U(q)[\exp (i q R)-$ $\left.\exp \left(i q R^{\prime}\right)\right]$. In the small $q$ limit, the difference of the exponential cancels out the singularity in the denominator of $U$.

[10] We have left out the $q=0$ term in the Fourier contribution in the Ewald sum for the electron impurity interaction and the electron-electron interaction because the system is electrically neutral.

[11] H. R. Ma and S. T. Chui, Phys. Rev. Lett. 67, 505 (1991).

[12] J. Jensen, A. Brass, A-C. Shi, and A. J. Berlinsky, Phys. Rev. B 41, 6394 (1990), and references therein.

[13] B. Tanatar and S. T. Chui, J. Phys. Condens. Matter 6, L485 (1994).

[14] There is a umklapp contribution from the shear mode, the logarithmic divergence of which was cut off due to the formation of domains. See, for example, the next reference. This contribution is reduced by the factor $\exp (-G d) \approx 0.05$ from $U(G)$ in the present case.

[15] M. Ferconi and G. Vignale, Phys. Rev. B 48, 2831 (1993).

[16] V.M. Pudalov, S. G. Semenchinskii, and V.S. Edelman, Zh. Eksp. Teor. Fiz. 89, 1870 (1985) [Sov. Phys. JETP 62, 1079 (1985); F. F. Fang (private communication).

[17] D. Stein, G. Ebert, K. von Klitzing, and G. Weimann, Surf. Sci. 142, 406 (1984).

[18] E.P. Wigner, Phys. Rev. 46, 1002 (1934).

[19] P. A. Lee and T. V. Ramakrishnan, Rev. Mod. Phys. 57, 287 (1985).

[20] J. Imry and S. K. Ma, Phys. Rev. Lett. 35, 1399 (1975).

[21] J.M. Kosterlitz and D. Thouless, J. Phys. C 7, 1046 (1974).

[22] N.F. Mott, Metal Insulator Transitions (Taylor and Francis, London, 1974). 